\title{
Reduced Achilles Tendon Stiffness Disrupts Calf Muscle Neuromechanics in Elderly Gait
}

\author{
Rebecca L. Krupenevich ${ }^{a}$ Owen N. Beck $^{\text {b, c }}$ Gregory S. Sawicki ${ }^{b, c}$ \\ Jason R. Franz ${ }^{\mathrm{a}}$ \\ a Joint Department of Biomedical Engineering, University of North Carolina at Chapel Hill and North Carolina State

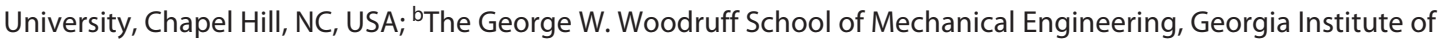 \\ Technology, Atlanta, GA, USA; 'The School of Biological Sciences, Georgia Institute of Technology, Atlanta, GA, USA
}

\section{Keywords}

Aging · Biomechanics · Stiffness · Energy expenditure ·

Muscle mechanics $\cdot$ Ankle exoskeleton

\section{Abstract}

Older adults walk slower and with a higher metabolic energy expenditure than younger adults. In this review, we explore the hypothesis that age-related declines in Achilles tendon stiffness increase the metabolic cost of walking due to less economical calf muscle contractions and increased proximal joint work. This viewpoint may motivate interventions to restore ankle muscle-tendon stiffness, improve walking mechanics, and reduce metabolic cost in older adults.

(c) 2021 S. Karger AG, Basel

\section{Introduction}

Older adults ( $\geq 65$ years) walk slower and, even when walking at the same speed, do so while consuming metabolic energy more quickly than younger adults (e.g., 1835 years) [1]. These changes are associated with difficulty in performing activities of daily living, and ultimately re- duce independence and quality of life. Age-related reductions in ankle mechanical power during the push off phase of walking appear consistently in the literature, and are often accompanied by compensatory increases in hip mechanical power [2-4] (i.e., a distal to proximal redistribution of mechanical power). This is notable because the muscles responsible for ankle push off power (i.e., triceps surae muscles) are critical for modulating walking speed [5], and indirect evidence suggests that ankle push off contributes to the metabolic cost of walking [6]. Accordingly, we posit that reduced ankle mechanical power at least partially contributes to the slower walking speed and increased energy consumption, which is observed in older adults.

Historically, efforts to increase ankle mechanical power have focused on increasing leg muscle strength. However, strength training interventions, while effective at increasing force-generating capacity, are generally ineffective at increasing ankle power and do not reduce metabolic energy consumption [7]. Given that ankle mechanical power is not solely generated by muscle output, and instead arises from the interaction between the triceps surae muscle and the Achilles tendon, these efforts may be misguided. In this review, we examine the over- 


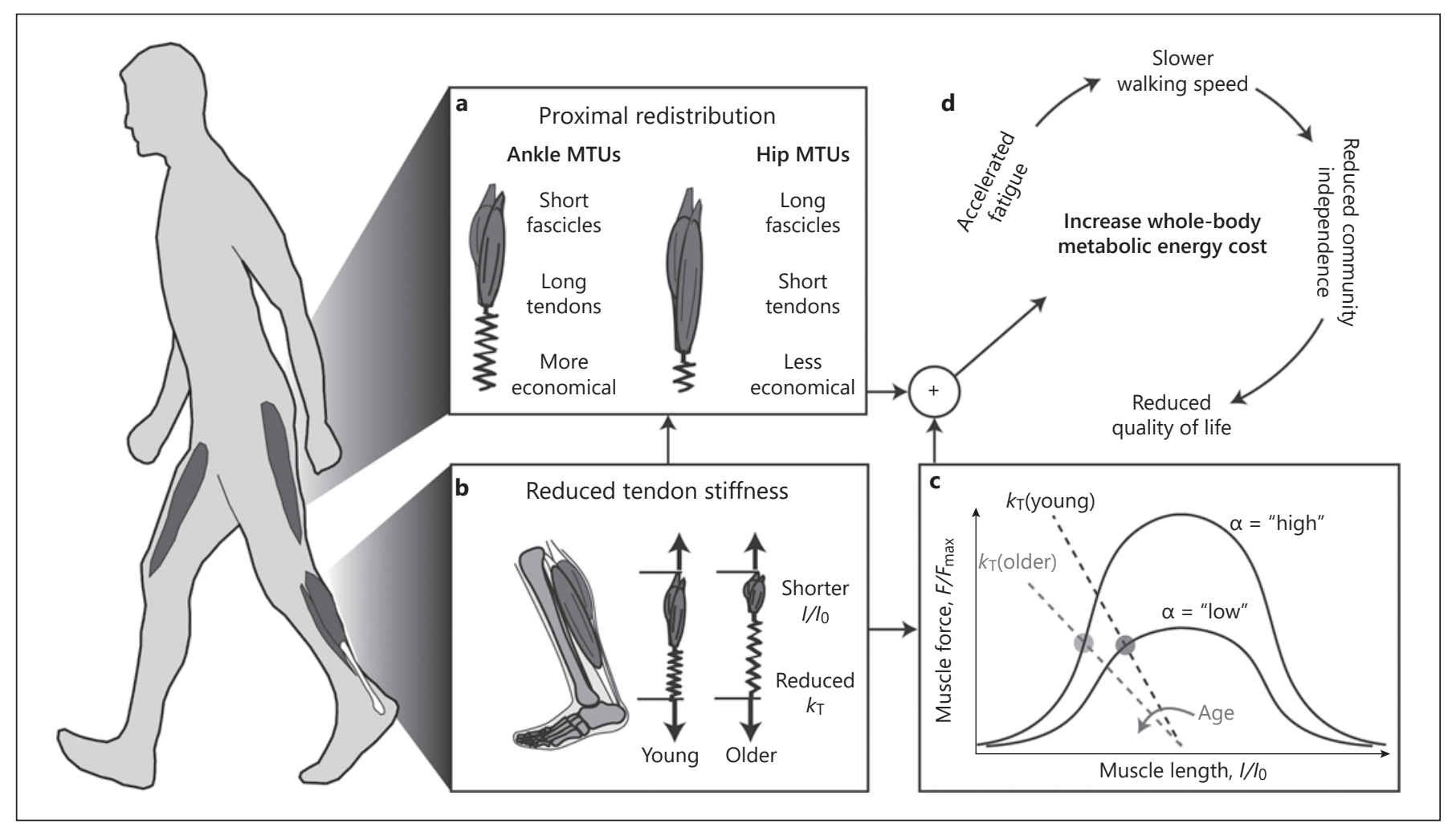

Fig. 1. We propose the novel hypothesis that an age-related decline in Achilles tendon stiffness $\left(k_{\mathrm{t}}\right)$ increases the metabolic cost of walking due to less economical triceps surae muscle contractions and increased proximal joint work (d). Conceptually, in order for older adults to maintain tuned ankle neuromechanical function to the level of young adults during walking (in the presence of reduced $k_{\mathrm{t}}$ ), they must operate at shorter triceps surae lengths and higher excitations (b); a metabolically costly adaptation that, due to reduced force-generating potential, yields lower force per unit

arching hypothesis that an age-related decline in Achilles tendon stiffness increases the metabolic cost of walking due to less economical triceps surae muscle contractions and increased proximal joint work (Fig. 1). We begin by summarizing our contemporary understanding of the neuromechanical interaction between active (triceps surae muscle) and passive (Achilles tendon) tissue mechanics and how this manifests as ankle mechanical power output during walking. We then discuss how age-related changes in Achilles tendon structure influence its stiffness and we propose a mechanistic pathway by which those changes yield biomechanical and metabolic consequences during walking in older adults. Finally, we describe clinical implications and suggestions derived from the core focus of our review, with specific regard to interventions focused on restoring ankle muscle-tendon struc- activation (c). Upper limits on triceps surae muscle recruitment (i.e., $100 \%$ activation) impose a threshold above which age-related decreases in Achilles tendon stiffness cannot be surmounted via the local adaptive response of increased triceps surae activation (a). Thus, older adults exhibit characteristic compensatory redistributions of mechanical workload to muscles spanning the hip which, due to differences in anatomical architecture compared to those spanning the ankle, further increase the metabolic cost of walking (a). tural stiffness to improve walking mechanics and energetics in aging, including translational opportunities for the bioinspired design and use of assistive devices.

\section{The Achilles Tendon Plays a Critical Role in Ankle Power Generation}

Recent advances in functional ultrasound imaging [8$10]$ and musculoskeletal modeling $[5,11]$ have elucidated the contribution of Achilles tendon recoil to ankle power output during walking. The passive viscoelastic Achilles tendon and the active triceps surae muscles interact to generate power via a "catapult" mechanism $[8,9,11]$. In the early stance phase of walking, triceps surae muscle fascicles generate force nearly isometrically, as the Achil- 
les tendon is simultaneously stretched while storing elastic energy. Then, in late stance, rapid shortening of the tendon allows the triceps surae muscle fascicles to remain at a relatively slower shortening velocity, promoting economical force generation (i.e., greater magnitude per unit activation) and thus propulsive power for ankle push off [8].

However, an effective ankle catapult is not guaranteed merely by the presence of the Achilles tendon; musculoskeletal models and simulations suggest that optimal ankle function requires a tuned interaction between active muscle stiffness and passive Achilles tendon stiffness [11, 12]. In this context, we distinguish muscle stiffness, defined as the localized slope of the length-tension curve [13], from short range stiffness, conventionally defined as the intrinsic stiffness of the fibers' myofilaments during isometric conditions. Short range stiffness does not account for stiffness modulation via neural control, and thus poorly predicts muscle stiffness during locomotion $[14,15]$. When the triceps surae muscles are actively stiffened in the early stance phase of walking, a clutch-like interaction is initiated engaging the fixed passive stiffness of the Achilles tendon that gives rise to a neuromechanical resonance $[16,17]$. In this case, the impedance of the source, a relatively stiff isometric muscle, is "tuned" to enable effective mechanical energy transfer from the center of mass to the elastic tissues within the triceps surae muscle-tendon unit and back again, driving a large portion of impulsive ankle push off via elastic recoil of the Achilles tendon $[9,18,19]$. Indeed, cumulative evidence suggests ankle muscle-tendon interaction is highly dependent on muscle-tendon properties, such as tendon stiffness and activation-dependent length-tension behavior of muscles.

\section{Age-Related Changes in Achilles Tendon Stiffness}

There is evidence to suggest that, in humans, Achilles tendon stiffness decreases with age [20-22]. Indeed, agerelated differences in Achilles tendon structural properties occur as early as middle age ( $\sim 45$ years) $[20,23]$. Onambele et al. [20] and Stenroth et al. [21] found 39 and $17 \%$ lower in vivo Achilles tendon stiffness in older (mean age 68 and 75 years) versus young adults (mean age 24 years), respectively (Fig. 2a). Previous studies have also reported indirect outcomes that are consistent with an age-related reduction in Achilles tendon stiffness. For example, even when walking with a similar ankle range of motion, Mian et al. [24] found shorter gastrocnemius fas-

Achilles Tendon Stiffness and Walking

Mechanics in Aging cicle length and greater Achilles tendon length in older versus young adults, and Panizzolo et al. [25] found that older adults exhibited shorter soleus fascicle lengths than younger adults - the results of both studies allude to a reduction in Achilles tendon stiffness in their older adult cohorts. More recently, we [26] revealed that Achilles tendon tissue displacements in older adults were, on average, $63 \%$ more sensitive to changes in tendon force than those in younger adults - an outcome consistent with age-related structural changes contributing to reduced Achilles tendon stiffness (Fig. 2b).

Nevertheless, there are some disparities in the literature with respect to age-related changes in tendon stiffness. Moreover, those changes may not be confined to the Achilles tendon. However, the implications of changes in tendon stiffness may be most functionally relevant for muscle-tendon units spanning the ankle due to their long tendons and greater reliance on tendon energy storage and return during functional activities. We also note that discrepancies in study outcomes exist within the human literature, with some studies reporting no difference in Achilles tendon stiffness between young and older adults $[27,28]$. Speculating about the reasons for these disparities is outside the scope of the present review. Despite the wealth of literature on this topic, there is no definitive consensus describing the effects of age on Achilles tendon stiffness in humans or animals (see online suppl. Fig. 1; see www.karger.com/doi/10.1159/000516910 for all online suppl. material). Longitudinal studies are ideal but often impractical way to understand aging effects of on walking mechanics and energetics. Cross-sectional designs that include larger sample sizes across a broad age range would be valuable for understanding the time course of how Achilles tendon stiffness and muscle-tendon dynamics change with age. In an effort to be fully transparent in our reporting of the literature regarding age-related changes in Achilles tendon stiffness, we summarize the outcomes of the contemporary literature in online suppl. Fig. 1.

\section{Muscular Response to Decreased Achilles Tendon Stiffness}

Based on available evidence, including the computational and experimental outcomes that follow, we consider it highly likely that reduced Achilles tendon stiffness in older adults disrupts the tuned neuromechanical interaction with the triceps surae during walking. Conceptually, age-related reductions in Achilles tendon stiffness 


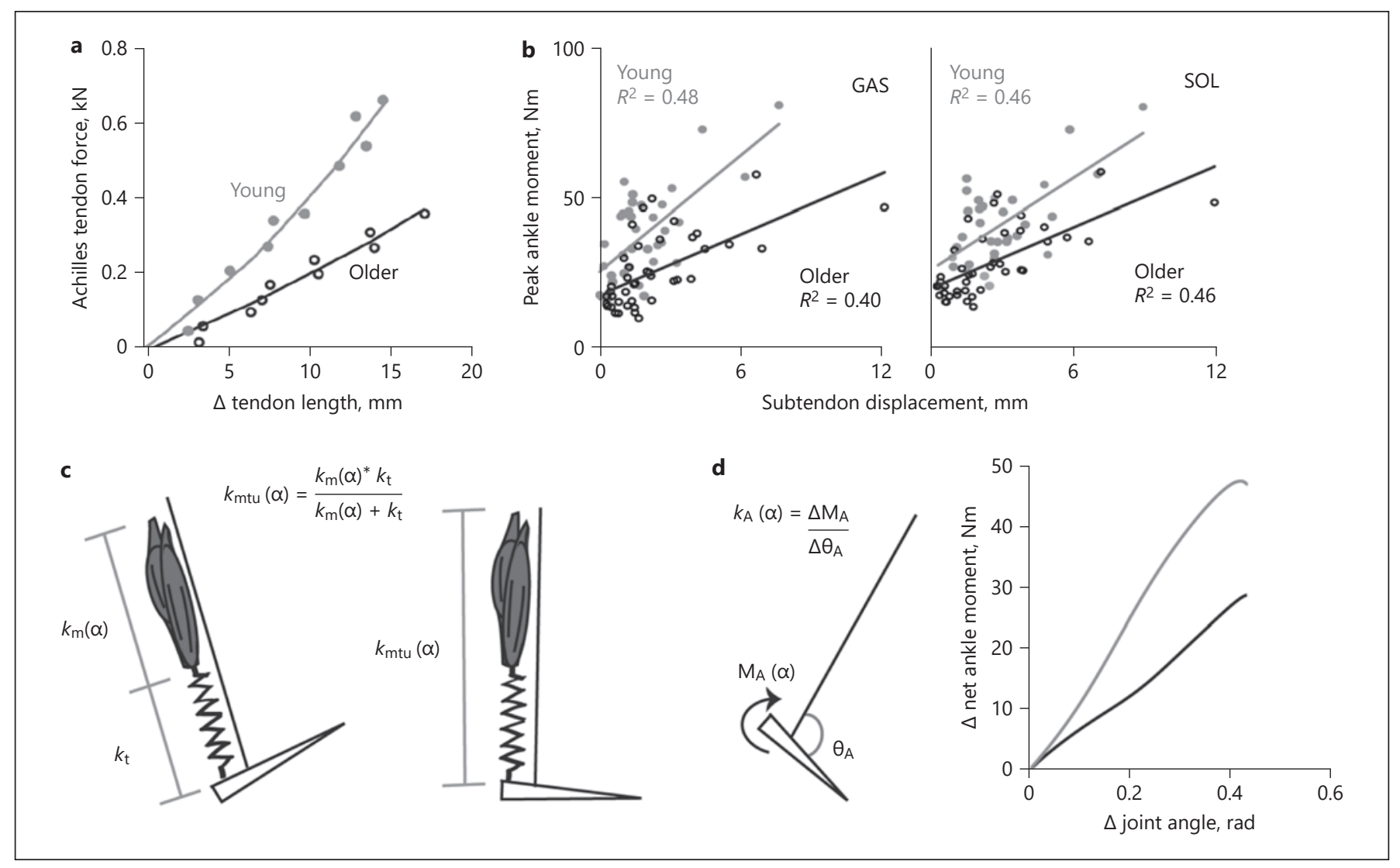

Fig. 2. a Achilles tendon stiffness given as the association between Achilles tendon force and Achilles tendon length in young (gray) and older (black) adults adapted from Onambele et al. [20]. b Correlations between peak net ankle moment and GAS and SOL subtendon tissue displacement in $n=9$ young (gray, closed circles) and $n=10$ older (black, open circles) adults, adapted from Clark and Franz [26]. c Illustrations depict the dependence of ankle muscletendon unit stiffness $\left(k_{\mathrm{mtu}}\right)$ on muscle activation $(\alpha)$, muscle stiffness

would elicit more tendon stretch for a given load. During walking, young and older adults exhibit similar ankle joint kinematics [2], and thus similar triceps surae muscle-tendon unit lengths [24]. Thus, for a given level of activation, we would expect a more compliant Achilles tendon in older adults to yield shorter triceps surae muscle fascicle operating lengths than those in younger adults (Fig. 1b, 3a). This premise is supported by walking simulations showing that to maintain the same ankle power output, lower Achilles tendon stiffness causes triceps surae muscle fascicles to operate at shorter lengths [12] (Fig. 3a). Although in vivo evidence to date is relatively limited, the 2 published experimental comparisons are consistent with these model predictions; gastrocnemius [24] and soleus [25] muscle fascicles operate at 10 and $\left(k_{\mathrm{m}}\right)$, and Achilles tendon stiffness $\left(k_{\mathrm{t}}\right)$. d Illustration depicts the dependence of ankle joint stiffness $\left(k_{\mathrm{A}}\right)$ on muscle activation, net ankle moment $\left(M_{\mathrm{A}}\right)$, and ankle angle $\left(\theta_{\mathrm{A}}\right)$. Plot shows ankle joint stiffness, given as the slope of net ankle moment and ankle angle, during isokinetic eccentric contractions of the triceps surae muscles at a matched activation of 75\% MVIC, prescribed via biofeedback, in $n=9$ young (gray) and $n=8$ older (black) adults, adapted from Krupenevich et al. [30]. GAS, gastrocnemius; SOL, soleus.

$16 \%$ shorter lengths in older than in younger adults during the stance phase of walking, respectively. Recent data from our group [29] are similar to the findings of prior studies and show $10 \%$ shorter medial gastrocnemius operating lengths in older versus young adults (Fig. 3b).

Here, we are operating under the assumption that relative fascicle lengths are shorter in older adults. Shorter operating lengths relative to optimal length due to reduced tendon stiffness would result in reduced force generation at submaximal activations and reduced forcegenerating capacity at maximum activations. Thus, to overcome the decreased force-generating potential associated with shorter fascicle operating lengths, older adults would need to increase muscle activation in order to maintain ankle muscle-tendon stiffness to the same level 
Fig. 3. a Simulated GAS and SOL excitation (top) and fiber length (bottom) plotted over an average stance phase as a function of Achilles tendon stiffness, adapted from Orselli et al. [12]. The small arrows indicate reduced Achilles tendon stiffness $\left(k_{\mathrm{t}}\right)$, and large arrows indicate the direction of change in excitation and fiber length with reduced $k_{\mathrm{t}}$. Thus, as $k_{\mathrm{t}}$ is reduced, GAS and SOL excitation increases, and fiber length decreases. b Average GAS fascicle length (adapted from Browne and Franz [29]) and peak SOL activation reported as a percent of maximum voluntary isometric contraction (i.e., \%MVC) (adapted from Franz and Kram [31]) during walking in young (gray) and older (black) adults. c SOL muscle stiffness, given by the relationship between SOL muscle force and SOL muscle length, in young adults during isokinetic eccentric contractions of the triceps surae muscles as a function of muscle activation ( $\alpha$ - indicated with the large arrow from $\alpha=0$ to $75 \%)$ prescribed via biofeedback, adapted from Clark and Franz [34]. d Model-predicted effects of peak Achilles tendon strain $\left(\varepsilon_{\mathrm{o}}\right)$ on GAS and SOL mass-normalized metabolic energy consumption. The small arrow indicates reduced $k_{\mathrm{t}}$, and the large arrow indicates the direction of change in SOL and GAS metabolic energy with reduced $k_{\mathrm{t}}$. GAS, gastrocnemius; SOL, soleus. a
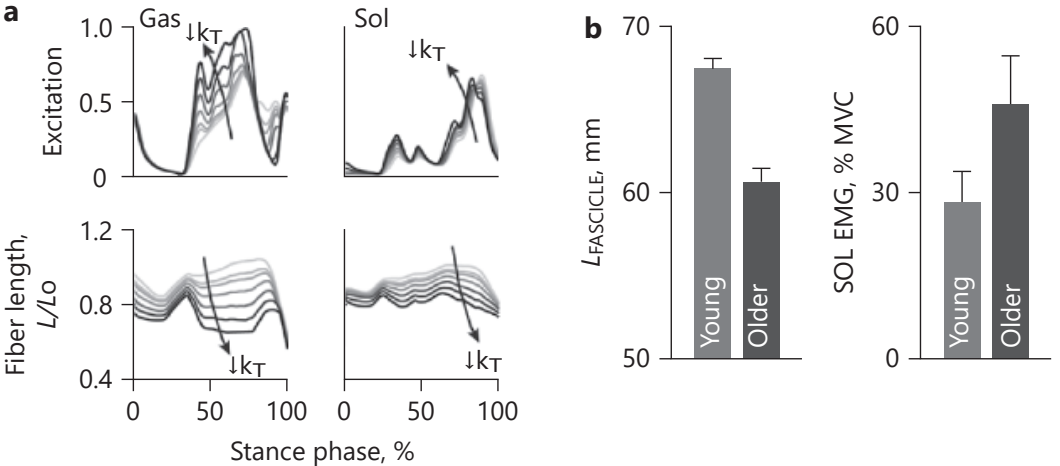

c
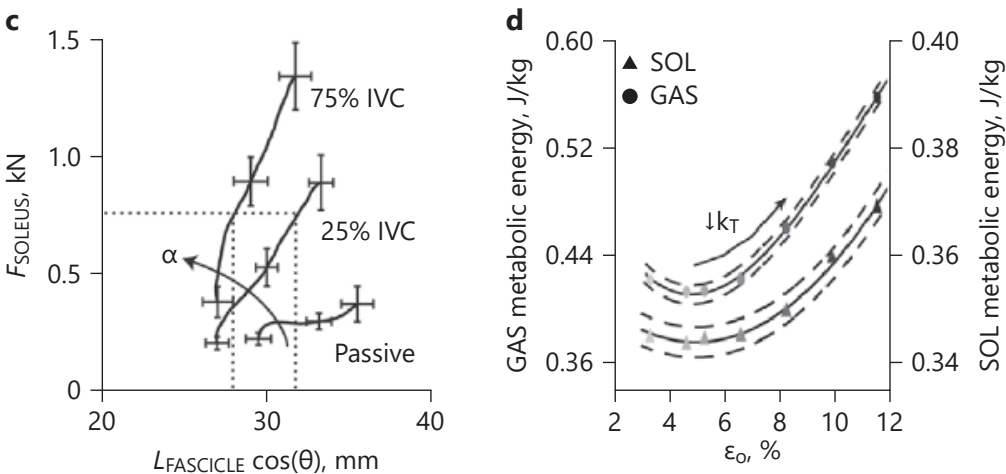

as younger adults [30]. Indeed, we previously reported that normalized stance phase soleus activity (i.e., \% maximum isometric activation) was $71 \%$ higher in older versus young adults [31] (Fig. 3b). Moreover, our simulations have shown that reductions in Achilles tendon stiffness (resulting in systematic increases in peak strain from 3.3 to $11.6 \%$ ) elicit progressively shorter triceps surae fascicle operating lengths and higher activation for identical task demands [12] (Fig. 3a). Increasing triceps surae muscle stiffness via elevated activation would, in part, serve to surmount the "structural bottleneck" (i.e., shorter muscle fiber lengths and resulting force-length decrement) imparted by a more compliant Achilles tendon, thus preserving overall muscle-tendon unit stiffness. Given the results of these simulations, we posit that when walking at matched speeds, older adults maintain overall muscletendon stiffness by shifting to higher triceps surae activation. This premise is supported by our recent findings that older adults display reduced triceps surae muscletendon stiffness, calculated as the slope of ankle moment and ankle angle, during isolated contractions at a matched activation level [30] (Fig. 2d), but similar triceps surae muscle-tendon stiffness during walking compared to younger adults. Although consistent with the findings of Crenna and Frigo [32], we note that our hypothesized mechanism contrasts with the findings of Stenroth et al. [33] who found that young and older adults displayed similar medial gastrocnemius fascicle length and muscletendon unit lengths during the stance phase of walking.

Although it is plausible that triceps surae muscle behavior can affect muscle-tendon unit stiffness via changes in activation, little empirical evidence exists for this interaction. To address this limitation, we recently combined in vivo ultrasound imaging with electromyographic biofeedback in young adults to quantify the extent to which ankle muscle-tendon stiffness can be modulated through neurally mediated changes in triceps surae muscle stiffness [34] (Fig. 3c). In a series of eccentric isokinetic ankle rotation tasks, subjects were either relaxed (i.e., passive) or matched their instantaneous soleus activation to target lines corresponding to 25 and $75 \%$ of their maximum isometric activation. We found that ankle muscle- 


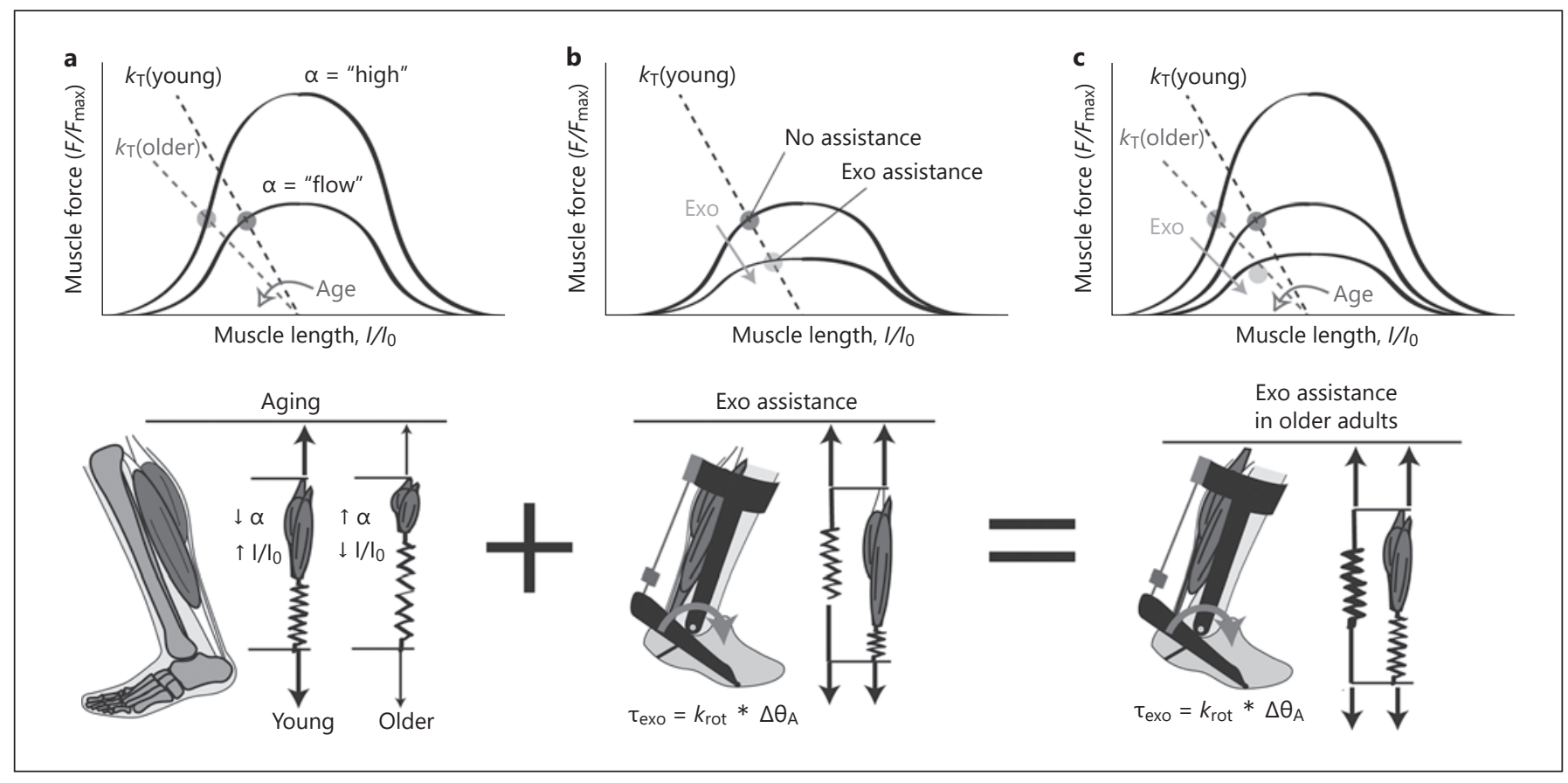

Fig. 4. Illustration of the proposed effect of elastic ankle exoskeleton assistance on mitigating age-related changes in triceps surae muscle length-tension behavior that arise from reduced Achilles tendon stiffness during walking. The left column (a) illustrates the hypothesized effect of age-related reductions in Achilles tendon stiffness $\left(k_{\mathrm{t}}\right)$ on muscle length-tension behavior, where reduced $k_{\mathrm{t}}$ in older adults results in shorter fascicle lengths $\left(l / l_{0}\right)$ and associated reductions in muscle force $\left(F / F_{\max }\right)$. The center column (b) illustrates the effect of exoskeleton assistance on muscle lengthtension behavior, where $\tau_{\text {exo }}$ indicates the extensor torque applied about the ankle from the exoskeleton, $k_{\text {exo }}$ indicates the rotational

tendon unit stiffness can be modulated via activation-dependent changes in soleus muscle stiffness. We also found that both soleus muscle stiffness and ankle muscle-tendon unit stiffness values exhibited nonlinear relationships with muscle activation. Muscle-tendon unit stiffness $\left(k_{\mathrm{mtu}}\right)$ can be approximated from muscle stiffness $\left(k_{\mathrm{m}}\right)$, as a function of activation $(\alpha)$, and tendon stiffness $\left(k_{\mathrm{t}}\right)$ according to

$$
k_{\mathrm{mtu}}(a)=\frac{k_{\mathrm{m}}(a) \times k_{t}}{k_{\mathrm{m}}(a)+k_{t}} .
$$

Figure $2 \mathrm{c}$ ankle joint stiffness $\left(k_{\mathrm{A}}\right)$ can be calculated as the slope of the relation between ankle joint moment $\left(\Delta M_{\mathrm{A}}\right)$ and ankle joint angle $\left(\Delta \theta_{\mathrm{A}}\right)$ and represents the aggregate stiffness of all passive and active structures crossing the ankle joint (Fig. 2d):

$$
k_{\mathrm{A}}(a)=\frac{\Delta M_{\mathrm{A}}}{\Delta \theta_{\mathrm{A}}} .
$$

stiffness of the exoskeleton, and $\Delta \theta_{\mathrm{A}}$ indicates the change in ankle angle. The exoskeleton behaves as a rotational spring $\left(\tau_{\text {exo }}=k_{\text {rot }} \times \Delta \theta_{\mathrm{A}}\right)$, adding structural stiffness to the biological triceps surae muscle-tendon units. The right column (c) illustrates the proposed effect of exoskeleton assistance in older adults. The exoskeleton acts to bypass age-related reductions in $k_{\mathrm{t}}$ via exoskeleton-applied torque $\left(\tau_{\text {exo }}\right)$, which effectively reduces the demand on the triceps surae muscle-tendon units and allows the triceps surae muscles to operate at longer lengths $\left(l / l_{0}\right)$ and lower activations $(\alpha)$.

In line with the relationships that govern stiffness of structural elements arranged in series (Eq. (1)), our data support the premise that ankle muscle-tendon unit stiffness arises from a combination of activation-independent components (i.e., the passive elastic Achilles tendon) and activation-dependent components (i.e., muscle) (Fig. 2). By extension, our data demonstrate how age-related reductions in Achilles tendon stiffness could be, at least in part, surmounted by increasing gastrocnemius and/or soleus muscle activation in order to maintain the stiffness of the ankle joint during walking (Eq. (2)). Unfortunately, our model predictions suggest that even if activation-dependent increases in muscle stiffness provide effective compensation for reduced Achilles tendon stiffness, it may come with a severe metabolic penalty [12] (Fig. 3d). 


\section{Metabolic Consequences of the Local Muscle Response}

The purported local adaptive response to reduced Achilles tendon stiffness - specifically, the shift to relatively shorter fascicle operating lengths and greater triceps surae muscle activation - has consequences (Fig. 1b). Modeling and simulation studies [12] suggest that walking with relatively greater triceps surae activation increases muscle metabolic energy expenditure. Namely, Orselli et al. [12] simulation study indicated that increasing Achilles tendon peak strain increased soleus and gastrocnemius muscle metabolic energy expenditure by 13 and $42 \%$, respectively (Fig. 3d). This increased muscle metabolic energy expenditure is consistent with previous simulation studies $[18,19]$. Thus, the local metabolic consequences (metabolic cost of operating individual muscles) associated with reduced Achilles tendon stiffness may be predominately due to increased triceps surae activation needed to overcome decreases in force-length and forcevelocity potential imposed by operating with shorter muscle fascicle lengths [35] (Fig. 1c, 3, 4a). Moving forward, empirical studies are warranted to confirm the findings of computational modeling studies, and to characterize the age-related adaptive response of the triceps surae muscles due to decreased Achilles tendon stiffness, and its effect on muscle metabolic energy expenditure.

Unfortunately, given upper limits on muscle recruitment, the muscle level adaptive response to increase triceps surae activation in the presence of lower Achilles tendon stiffness may only be partially effective at preserving gross triceps surae muscle-tendon unit behavior. Indeed, we believe that reduced Achilles tendon stiffness not only increases local metabolic energy expenditure (Fig. 1c), but that it also facilitates an unfavorable cascade of biomechanical and metabolic adaptations within the limb (Fig. 1a). As we describe in the next section, this cascade could serve as a mechanistic framework for linking age-related reductions in ankle mechanical power output and the subsequent compensatory redistribution of mechanical work to the hip during walking; a strategy that, while biomechanically effective, may increase the wholebody metabolic energy consumption during walking.

\section{Metabolic Consequences of the Limb Level Response}

We have so far presented data to suggest that triceps surae muscle activation can modulate ankle muscletendon unit stiffness (Eq. 1) and thereby modulate an- kle joint mechanics (Eq. 2) (Fig. 3). However, upper limits on triceps surae muscle recruitment impose a threshold above which age-related decreases in Achilles tendon stiffness cannot be surmounted. Indeed, older adults exhibit hallmark reductions in ankle joint moment and ankle push off power $[2,4,36]$, that are often accompanied by increased hip mechanical power. The increased reliance of leg muscles spanning the hip for power generation is thought to be a compensatory response for the well-documented deficits in ankle power output $[4,36]$. The muscle-tendon architecture of hip muscles (e.g., with longer fascicles and short tendons) may be less favorable for economic force production compared to that of ankle muscles (e.g., with shorter fascicles and long tendons) [37](Fig. 1a). Activating longer muscle fascicles yields a greater volume of actinmyosin cross bridges, and require more metabolic energy to generate a unit of fascicle force [38]. Thus, as hypothesized, an age-related decline in Achilles tendon stiffness may increase the metabolic cost of walking due to less economical triceps surae muscle contractions as well as increased proximal joint work. Furthermore, the whole-body metabolic penalties of these compensations to surmount the "structural bottleneck" imparted by a more compliant Achilles tendon may ultimately prompt older adults to succumb to slower preferred walking speeds [33].

We acknowledge that age-related changes in walking mechanics are complex and multifactorial. In fact, several previous review papers have explored alternative explanations for these changes $[39,40]$. For example, it has been suggested that increased forward trunk lean contributes to the age-related increase in hip mechanical power [3]. However, recent evidence suggests no change in hip mechanics in older adults walking with a more upright trunk compared to a self-selected trunk lean [41]. Here, we seek to move the literature forward by focusing specifically on age-related changes in the neuromechanics of the ankle muscle-tendon units, which have been repeatedly implicated with slower walking speeds and increased metabolic energy consumption $[1,4]$. Establishing a link between age-related changes in the structural properties of ankle muscletendon units and elevated energy cost of locomotion will provide leverage for interventions that aim to restore or improve mobility in our aging population. In the next sections we explore current and future directions in exercise, exoskeletons, and regenerative robotic interventions. 


\section{Can Older Adults Outrun Compliant Tendons?}

If an age-related decrease in Achilles tendon stiffness disrupts triceps surae neuromechanical function, perhaps older adults who maintain youthful Achilles tendon stiffness also preserve walking biomechanics and metabolic energy cost. A factor with some demonstrated efficacy to attenuate age-related deteriorations in both Achilles tendon stiffness and walking economy is distance running exercise. Separate cross-sectional studies have reported that older adults who run for exercise (older runners) have similar mass-specific Achilles tendon stiffness [28, 42] and walking economy values [43] as young adults. These studies also reported that older runners exhibited 10-16\% stiffer mass-specific Achilles tendons $[28,42]$ and $7-10 \%$ superior walking economy values [43] than age-matched older non-runners, suggesting that older adults may be able to "outrun" age-related reductions in Achilles tendon stiffness and increased metabolic energy expenditure. Vigorous walking programs have also shown a positive effect on the metabolic cost of walking and preferred walking speed in older adults $[44,45]$. However, the mechanisms underlying these changes are unknown. Longitudinal studies that comprehensively examine changes in Achilles tendon stiffness, walking biomechanics, and metabolic cost in both young and older adults who participate in running or walking programs are needed to confirm these crosssectional observations. There is also evidence to suggest that resistance training may increase Achilles tendon stiffness in older adults [46]. Therefore, training programs that combine resistance exercise and walking or running may be efficacious at improving or maintaining mobility in older adults.

\section{Exoskeletons to Augment Older Adult Mobility}

Even if distance running exercise maintains or restores youthful Achilles tendon stiffness and thereby stifles the cascade of events hypothesized to follow, it may not be feasible for many adults to begin a distance running program in the seventh decade of life. Similarly, although vigorous walking interventions may improve the metabolic cost of walking and increase walking speed, many older adults may be limited by their current functional abilities. Assistive devices, such as ankle exoskeletons, may provide a more feasible approach to supplement older adult ankle-joint function and walking economy. Exoskeletons are wearable devices that are engineered to alter limb joint biomechanics and augment human performance [47]. A subset of these devices is unpowered, and do not require an external power source, but instead provide assistive torque using elastic elements that provide rotational stiffness in parallel to the biological limb joint (e.g., Fig. 4b). Today, research quality elastic ankle exoskeletons have the ability to reduce metabolic cost of walking by up to $7 \%$ in young adults $[48,49]$ by attenuating triceps surae muscle-tendon force and active muscle volume [35]. Within the triceps surae muscle-tendon units, lower forces lead to reduced Achilles tendon strain and increased triceps surae fascicle operating lengths (Fig. 4b). Specifically, elastic ankle exoskeletons have been reported to increase young adult soleus fascicle operating lengths by up to $11 \%$ at the time of peak soleus force generation [50], which is roughly equivalent to the reported age-related reduction in mean normalized fascicle lengths during the stance phase of walking [25]. Thus, the available muscle level data from young adults walking with elastic exoskeletons suggest that exoskeletons have the exact opposite effect (Fig. 4b) that the age-related loss of Achilles tendon stiffness (Fig. 4a) does on triceps surae muscle dynamics. Hence, by externally adding structural stiffness in parallel with the triceps surae muscles, elastic ankle exoskeletons have the potential to mitigate both local (Fig. 1c, 4c) and global (Fig. 1a) metabolic penalties associated with age-related reductions in Achilles tendon stiffness (Fig. 1b, 2). We note that the studies discussed above only refer to the soleus muscle with the assumption that the gastrocnemius would behave similarly in response to exoskeleton assistance. However, it is possible that differences in gastrocnemius function and architecture result in different behavior. There is some evidence to suggest that exoskeletons targeting the gastrocnemius response may actually yield a greater reduction in the metabolic cost of walking compared to exoskeletons targeting the soleus response [51].

Applying exoskeletons to older adult ankles are not a new concept [52, 53]. For instance, Galle et al. [53] presented stride kinematics and metabolic data from older adults walking with and without a tethered ankle exoskeleton that injected external mechanical energy to power push off. Compared to not using a device, their older participants increased step length when using the exoskeleton, but walking economy only "improved" by a nonstatistically significant 4\% [53]. Furthermore, in that study, older adult walking economy improved less than that of younger adults using the same device and controller [53]. The dissimilar metabolic response between these cohorts 
suggests that optimal ankle exoskeleton parameters may differ between young and older adults. One potential explanation is underlying differences in Achilles tendon stiffness. For example, to compensate for their relatively compliant morphology, an unpowered ankle exoskeleton using elastic elements to provide structural support may need to provide relatively more structural stiffness for an older adult user than a younger adult user. In addition, differences in performance between young and older exoskeleton users could also stem from age-related differences in muscle metabolism. Older adults exhibit decreased mitochondrial density and efficiency compared to younger adults[54], which may serve as a limiting factor for exoskeleton-related metabolic improvements in older adults. On the other hand, physical activity-matched young and older adults do not display differences in mitochondrial efficiency, suggesting that some of the agerelated differences in muscle metabolism reported in the literature may be due to age-related declines in physical activity and not age, per se [55]. Thus, although researchers have begun to converge on the optimal ankle exoskeleton assistance profile(s) for young adults [56-58], parameters may need to be updated to maximize locomotion performance for older users. Future studies are needed to confirm the effects of exoskeleton assistance on triceps surae behavior and metabolic energy expenditure in older adults.

\section{Regenerative Robotics - Advanced Technology to Maintain Tissue Health}

Advancements in technology and medicine may spark alternative opportunities for augmenting muscle-tendon dynamics in our aging population. For example, exoskeleton controllers with access to biomarkers of tissue degeneration could modify their assistance parameters in response to material properties of user connective tissues (i.e., Achilles tendon). Alternatively, surgical interventions that adjust Achilles tendon slack length and restore length-tension properties are common after Achilles tendon rupture [59]. As these surgeries become less invasive, similar interventions may offer an opportunity to preserve walking economy for older adults. Emerging evidence also suggests that injections of lubricin or hyaluronic acid may help to maintain or restore tendon function with age. Optimistically, as the fields of tissue biomechanics, regenerative medicine, and wearable robotics continue to advance, the potential for regenerative robotics may be realized. Such advanced wearable systems, by

Achilles Tendon Stiffness and Walking Mechanics in Aging working to continuously adapt the mechanical and chemical environment surrounding tissues in vivo, could slow or prevent loss of tendon stiffness over the lifespan and improve quality of life by preserving mobility for our aging population.

\section{Alternative Viewpoints}

It is possible that age-related factors other than or in addition to reduced Achilles tendon stiffness disrupt triceps surae muscle-tendon interaction and these alternatives may have metabolic consequences. For example, older adults often exhibit increased coactivation of antagonist muscles spanning the ankle joint (e.g., tibialis anterior), a change that may serve to increase ankle joint stiffness during walking. Although this may offset reductions in Achilles tendon stiffness at the joint level, it also comes with metabolic penalties [60]. We also wish to restate the need for individual length-tension curves in young and older adults to determine the extent to which shorter fascicle lengths are driven by muscle architecture versus tendon stiffness. Additionally, although evidence generally supports reduced Achilles tendon stiffness with age, not all older adults are guaranteed to exhibit the same response. Screening practices may be necessary to guide the prescription of, for example, ankle exoskeleton assistance. However, since exoskeletons replace rather than augment triceps surae function, even older adults without reductions in Achilles tendon stiffness are likely to experience metabolic benefits.

\section{Conclusion}

Our aging population is experiencing dramatic declines in mobility, independence, and quality of life that, in part, stem from changes in structural properties of musculoskeletal tissues. In this review, we focus on the elevated energy cost of walking in older adults. We examine the novel hypothesis that an age-related decline in Achilles tendon stiffness presents a "structural bottleneck" that increases the metabolic cost of walking due to less economical triceps surae muscle contractions and increased proximal joint work. The metabolic penalty associated with these unfavorable biomechanical and metabolic adaptations may discourage activity with compounding impact on mobility and ultimately, happiness. Moving forward, there is a critical need to improve the quality of life in advanced age by addressing these func- 
tional declines, potentially through innovations in assistive device technology aimed at restoring musculoskeletal structure to maintain locomotor function over the lifespan.

\section{Conflict of Interest Statement}

The authors have no conflicts of interest to declare.

\section{Funding Sources}

This work was supported by grants from the National Institutes of Health (R01AG058615 to J.R.F. and G.S.S., F32AG063460 to O.N.B., and F32AG067675 to R.L.K.).

\section{Author Contributions}

All authors participated in drafting and revising this manuscript.

\section{References}

1 Mian OS, Thom JM, Ardigò LP, Narici MV, Minetti AE. Metabolic cost, mechanical work, and efficiency during walking in young and older men. Acta Physiol. 2006;186(2):127-39.

2 Kerrigan DC, Todd MK, Della Croce U, Lipsitz LA, Collins JJ. Biomechanical gait alterations independent of speed in the healthy elderly: evidence for specific limiting impairments. Arch Phys Med Rehabil. 1998 Mar; 79(3):317-22.

3 DeVita P, Hortobagyi T. Age causes a redistribution of joint torques and powers during gait. J Appl Physiol. 2000 May;88(5):1804-11.

4 Franz JR. The age-associated reduction in propulsive power generation in walking. Exerc Sport Sci Rev. 2016;44(4):129-36.

5 Neptune RR, Clark DJ, Kautz SA. Modular control of human walking: a simulation study. J Biomech. 2009;42(9):1282-7.

6 Huang TW, Shorter KA, Adamczyk PG, Kuo AD. Mechanical and energetic consequences of reduced ankle plantar-flexion in human walking. J Exp Biol. 2015;218(Pt 22):3541.

7 Mian OS, Thom JM, Ardigò LP, Morse CI, Narici MV, Minetti AE. Effect of a 12-month physical conditioning programme on the metabolic cost of walking in healthy older adults. Eur J Appl Physiol. 2007;100(5):499_ 505.

8 Ishikawa M, Komi PV, Grey MJ, Lepola V, Bruggemann GP. Muscle-tendon interaction and elastic energy usage in human walking. J Appl Physiol. 2005;99(2):603-8.

9 Lichtwark GA, Wilson AM. Effects of series elasticity and activation conditions on muscle power output and efficiency. J Exp Biol. 2005; 208(15):2845-53.

10 Franz JR, Thelen DG. Depth-dependent variations in Achilles tendon deformations with age are associated with reduced plantarflexor performance during walking. J Appl Physiol. 2015;119(3):242-9.

11 Zelik KE, Huang TW, Adamczyk PG, Kuo $\mathrm{AD}$. The role of series ankle elasticity in bipedal walking. J Theor Biol. 2014;346:75-85.

12 Orselli MIV, Franz JR, Thelen DG. The effects of Achilles tendon compliance on triceps surae mechanics and energetics in walking. J Biomech. 2017;60:227-31.
13 Walmsley B, Proske U. Comparison of stiffness of soleus and medial gastrocnemius muscles in cats. J Neurophysiol. 1981 Aug;46(2): 250-9.

14 de Vlugt E, van Eesbeek S, Baines P, Hilte J, Meskers CG, de Groot JH. Short range stiffness elastic limit depends on joint velocity. J Biomech. 2011 Jul 28;44(11):2106-12.

15 Sartori M, Maculan M, Pizzolato C, Reggiani $\mathrm{M}$, Farina D. Modeling and simulating the neuromuscular mechanisms regulating ankle and knee joint stiffness during human locomotion. J Neurophysiol. 2015 Oct;114(4): 2509-27.

16 Robertson BD, Sawicki GS. Exploiting elasticity: modeling the influence of neural control on mechanics and energetics of ankle muscletendons during human hopping. J Theor Biol. 2014 Jul 21;353:121-32.

17 Robertson BD, Sawicki GS. Unconstrained muscle-tendon workloops indicate resonance tuning as a mechanism for elastic limb behavior during terrestrial locomotion. Proc Natl Acad Sci U S A. 2015 Oct 27;112(43):E58918.

18 Lichtwark GA, Wilson AM. Is Achilles tendon compliance optimised for maximum muscle efficiency during locomotion? J Biomech. 2007;40(8):1768-75.

19 Lichtwark GA, Wilson AM. Optimal muscle fascicle length and tendon stiffness for maximising gastrocnemius efficiency during human walking and running. J Theor Biol. 2008 Jun 21;252(4):662-73.

20 Onambele GL, Narici MV, Maganaris CN. Calf muscle-tendon properties and postural balance in old age. J Appl Physiol. 2006; 100(6):2048-56.

21 Stenroth L, Peltonen J, Cronin NJ, Sipilä S, Finni T. Age-related differences in Achilles tendon properties and triceps surae muscle architecture in vivo. J Appl Physiol. 2012; 113(10):1537-44.

22 Csapo R, Malis V, Hodgson J, Sinha S. Agerelated greater Achilles tendon compliance is not associated with larger plantar flexor muscle fascicle strains in senior women. J Appl Physiol. 2014;116(8):961-9.
23 Slane LC, Thelen DG. Achilles tendon displacement patterns during passive stretch and eccentric loading are altered in middle-aged adults. Med Eng Phys. 2015;37(7):712-6.

24 Mian OS, Thom JM, Ardigò LP, Minetti AE, Narici MV. Gastrocnemius muscle-tendon behaviour during walking in young and older adults. Acta Physiol. 2007;189(1):57-65.

25 Panizzolo FA, Green DJ, Lloyd DG, Maiorana AJ, Rubenson J. Soleus fascicle length changes are conserved between young and old adults at their preferred walking speed. Gait Posture. 2013;38(4):764-9.

26 Clark WH, Franz JR. Triceps surae musclesubtendon interaction differs between young and older adults. Connect Tissue Res. 2019; 61:1-10.

27 Karamanidis K, Arampatzis A, Mademli L. Age-related deficit in dynamic stability control after forward falls is affected by muscle strength and tendon stiffness. J Electromyogr Kinesiol. 2008;18(6):980-9.

28 Stenroth L, Cronin NJ, Peltonen J, Korhonen MT, Sipilä S, Finni T. Triceps surae muscletendon properties in older endurance- and sprint-trained athletes. J Appl Physiol. 2015; 120(1):63-9.

29 Browne MG, Franz JR. Increasing gastrocnemius activity during walking may elicit counterproductive effects on fascicle behavior in older adults. Int Soc Biomech. 2019.

30 Krupenevich RL, Clark WH, Sawicki GS, Franz JR. Older adults overcome reduced triceps surae structural stiffness to preserve ankle joint quasi-stiffness during walking. J Appl Biomech. 2020;1:1-8.

31 Franz JR, Kram R. How does age affect leg muscle activity/coactivity during uphill and downhill walking? Gait Posture. 2013;37(3): 378-84.

32 Crenna P, Frigo C. Dynamics of the ankle joint analyzed through moment-angle loops during human walking: gender and age effects. Hum Move Sci. 2011;30(6):1185-98.

33 Stenroth L, Sipilä S, Finni Juutinen T, Cronin $\mathrm{N}$. Slower walking speed in older men improves triceps surae force generation ability. Med Sci Sport Exerc. 2017;49:158-66. 
34 Clark WH, Franz JR. Activation-dependent changes in soleus length-tension behavior augment ankle joint quasi-stiffness. J Appl Biomech. 2019;35:182-9.

35 Beck ON, Punith LK, Nuckols RW, Sawicki GS. Exoskeletons improve locomotion economy by reducing active muscle; 2019.

36 DeVita P, Hortobagyi T. Age causes a redistribution of joint torques and powers during gait. J Appl Physiol. 2000;88:1804-11.

37 Sawicki GS, Lewis CL, Ferris DP. It pays to have a spring in your step. Exerc Sport Sci Rev. 2009;37(3):130.

38 Roberts TJ, Chen MS, Taylor CR. Energetics of bipedal running. II. Limb design and running mechanics. J Exp Biol. 1998;201(19): 2753-62.

39 McGibbon CA. Toward a better understanding of gait changes with age and disablement: neuromuscular adaptation. Exerc Sport Sci Rev. 2003;31(2):102-8.

40 Sorenson SC, Flanagan SP. Age冈related changes to composite lower extremity kinetics and their constituents in healthy gait: a perspective on contributing factors and mechanisms. Healthy Aging Res. 2015;4:1-9.

41 Krupenevich RL, Miller RH. Effects of selfselected step length and trunk position on joint kinetics in highly physically fit older adults. J Appl Biomech. 2020;1:1-7.

42 Karamanidis K, Arampatzis A. Mechanical and morphological properties of human quadriceps femoris and triceps surae muscletendon unit in relation to aging and running. J Biomech. 2006;39(3):406-17.

43 Ortega JD, Beck ON, Roby JM, Turney AL, Kram R. Running for exercise mitigates agerelated deterioration of walking economy. PloS One. 2014;9(11):e113471.
44 Thomas EE, De Vito G, Macaluso A. Speed training with body weight unloading improves walking energy cost and maximal speed in 75- to 85-year-old healthy women. J Appl Physiol. 2007;103(5):1598-603.

45 Malatesta D, Simar D, Ben Saad H, Préfaut C, Caillaud C. Effect of an overground walking training on gait performance in healthy 65 - to 80-year-olds. Exp Gerontol. 2010;45(6):42734.

46 McCrum C, Leow P, Epro G, König M, Meijer $\mathrm{K}$, Karamanidis K. Alterations in leg extensor muscle-tendon unit biomechanical properties with ageing and mechanical loading. Front Physiol. 2018;9:150.

47 Sawicki GS, Beck ON, Kang I, Young AJ. The exoskeleton expansion: improving walking and running economy. J Neuroeng Rehabil. 2020;17(1):25-9.

48 Collins SH, Wiggin MB, Sawicki GS. Reducing the energy cost of human walking using an unpowered exoskeleton. Nature. 2015; 522(7555):212

49 Nuckols RW, Sawicki GS. Impact of elastic ankle exoskeleton stiffness on neuromechanics and energetics of human walking across multiple speeds. J Neuroeng Rehabil. 2020; 17(1):75-19.

50 Nuckols RW, Dick TJM, Beck ON, Sawicki GS. Ultrasound imaging links soleus muscle neuromechanics and energetics during human walking with elastic ankle exoskeletons. Sci Rep. 2020;10(1):3604-15.

51 Malcolm P, Galle S, Derave W, De Clercq D. Bi-articular knee-ankle-foot exoskeleton produces higher metabolic cost reduction than weight-matched mono-articular exoskeleton. Front Neurosci. 2018;12:69.
52 Norris JA, Granata KP, Mitros MR, Byrne EM, Marsh AP. Effect of augmented plantarflexion power on preferred walking speed and economy in young and older adults. Gait Posture. 2007;25(4):620-7.

53 Galle S, Derave W, Bossuyt F, Calders P, Malcolm P, De Clercq D. Exoskeleton plantarflexion assistance for elderly. Gait Posture. 2017; 52:183-8

54 Casuso RA, Huertas JR. The emerging role of skeletal muscle mitochondrial dynamics in exercise and ageing. Ageing Res Rev. 2020;58: 101025.

55 Brierley EJ, Johnson MA, James OF, Turnbull DM. Effects of physical activity and age on mitochondrial function. QJM. 1996;89(4): 251-8.

56 Jackson RW, Collins SH. An experimental comparison of the relative benefits of work and torque assistance in ankle exoskeletons. J Appl Physiol. 2015;119(5):541-57.

57 Nuckols RW, Collins SH, Sawicki GS. An emulator system to characterize optimal elastic ankle exoskeleton stiffness during human walking and running. Columbus, $\mathrm{OH}$ : Dynamic Walking Conf; 2015.

58 Zhang J, Fiers P, Witte KA, Jackson RW, Poggensee KL, Atkeson CG, et al. Human-in-theloop optimization of exoskeleton assistance during walking. Science. 2017;356(6344): $1280-4$

59 Lui TH. Endoscopic Achilles tendon shortening. Foot. 2018;35:5-10.

60 Hortobágyi T, Finch A, Solnik S, Rider P, DeVita P. Association between muscle activation and metabolic cost of walking in young and old adults. J Gerontol A Biol Sci Med Sci. 2011;66(5):541-7. 